\title{
Serum osteopontin is a predictor of prognosis for HBV-associated acute-on-chronic liver failure
}

\author{
LONGGEN LIU ${ }^{1,2^{*}}$, JIANCHUN LU ${ }^{1,2^{*}}$, CHUNYAN YE ${ }^{1,2}$, LIN LIN $^{1,3}$, \\ SHUQIN ZHENG ${ }^{1,2}$, HONGYU ZHANG ${ }^{1,2}$, QING LAN $^{4}$ and YUAN XUE ${ }^{1,2}$ \\ ${ }^{1}$ Institute for the Study of Liver Diseases; Departments of ${ }^{2}$ Liver Diseases and ${ }^{3}$ Pharmacy, \\ The Third People's Hospital of Changzhou, Changzhou, Jiangsu 213000; ${ }^{4}$ Department of Infectious Diseases, \\ The First Affiliated Hospital of Kunming Medical University, Kunming, Yunnan 650051, P.R. China
}

Received August 22, 2017; Accepted November 11, 2017

DOI: $10.3892 /$ br.2017.1027

\begin{abstract}
Acute-on-chronic liver failure (ACLF) is a syndrome with a high rate of short-term mortality, and clinically it is important to identify patients at high risk of mortality. The present study evaluated the value of osteopontin (OPN) in the prediction of 90-day mortality in patients with ACLF. A total of 54 patients with HBV-associated ACLF were enrolled, and serum OPN levels were determined in a prospective, observational study design. Survival analysis was performed using Kaplan-Meier curves, and multivariate Cox proportional hazards regression was used to analyze independent risk factors of mortality. Serum OPN was significantly higher in HBV-ACLF patients compared with patients with chronic hepatitis B and healthy controls (both $\mathrm{P}<0.01$ ), and furthermore, was higher in those patients who succumbed to HBV-ACLF compared with surviving patients $(\mathrm{P}<0.05)$. OPN level positively correlated with total bilirubin $(\mathrm{r}=0.554, \mathrm{P}<0.001)$, Model for End-Stage Liver Disease (MELD) score $(r=0.234, P=0.038)$, MELD-Na score $(r=0.379$, $\mathrm{P}=0.005)$ and monocyte count $(\mathrm{r}=0.282, \mathrm{P}=0.039)$, and OPN was an independent risk factor for 90-day mortality in
\end{abstract}

Correspondence to: Dr Yuan Xue, Institute for the Study of Liver Diseases, The Third People's Hospital of Changzhou, 300 Lanling North Road, Changzhou, Jiangsu 213000, P.R. China

E-mail: xueyuan80908@163.com

*Contributed equally

Abbreviations: HBV, hepatitis B virus; ACLF, acute-on-chronic liver failure; LC, liver cirrhosis; $\mathrm{CHB}$, chronic hepatitis B; OPN, osteopontin; HPC, hepatic progenitor cell; HCC, hepatocellular carcinoma; HSC, hepatic stellate cell; MELD, model for end-stage liver disease; ALT, alanine aminotransferase; AST, aspartate aminotransferase; TBil, total bilirubin; INR, international normalized ratio; WBC, white blood cell; AFP, $\alpha$-fetoprotein; r, correlation coefficient; CI, confidence interval; $\mathrm{HC}$, healthy control; OR, odds ratio

Key words: osteopontin, acute-on-chronic liver failure, prognosis, survival prediction, biomarker
ACLF $(\mathrm{P}=0.021$, odds ratio $=1.104,95 \%$ confidence interval: 1.003-1.116). Furthermore, ACLF patients were stratified into three groups according to serum OPN levels (low mortality risk: $<6,135 \mathrm{ng} / \mathrm{ml}$; intermediate risk: 6,135-9,043 ng/ml; and high risk: $>9,043 \mathrm{ng} / \mathrm{ml})$, for which the 90 -day mortality rates were 27.78 (5/18), 52.94 (9/17) and 73.68\% (14/19), respectively, and those in the high risk had a poorer prognosis compared with the low risk group $(\mathrm{P}=0.009)$. In conclusion, serum OPN may be an independent risk factor associated with HBV-ACLF prognosis.

\section{Introduction}

Hepatitis B virus (HBV)-associated acute-on-chronic liver failure (ACLF), characterized by acute exacerbation of chronic hepatitis $\mathrm{B}(\mathrm{CHB})$ or liver cirrhosis (LC), is associated with a high rate of short-term mortality (1-3). Thus, it is important to stratify the patients with poor prognosis and determine the degree of emergency for liver transplantation. Although a number of scoring systems, including the Model for End-Stage Liver Disease (MELD) (4) and MELD-Na (5) schema, are generally used for evaluating patients with HBV-ACLF, they have limited accuracy in predicting prognosis, and there is currently no effective model for predicting the prognosis of patients with ACLF (1).

Osteopontin (OPN), a secreted phosphorylated glycoprotein, may participate in the progression of liver injury by promoting the inflammatory response that induces macrophage and neutrophil activation and migration (6,7). A number of studies have suggested that OPN may serve as a biomarker of severe fibrosis and portal hypertension during Schistosomiasis mansoni (8) and chronic viral hepatitis $(9,10)$. Nevertheless, in mice models of liver injury induced by diethylnitrosamine or ischemia/reperfusion, OPN exerted a protective effect by ameliorating the production of IL-6 and TNF- $\alpha$ in macrophages and inhibiting inflammation $(11,12)$. Furthermore, OPN may serve a key role in the expansion of hepatic progenitor cells (HPC) and in promoting liver regeneration during liver cancer $(13,14)$ and acute liver failure $(15,16)$.

Thus, as an acute phase protein, OPN may serve critical roles in the inflammatory response and liver regeneration, though is considered to exert detrimental effect in liver 
injury. In addition, Arai et al (17) demonstrated that OPN was expressed in inflammatory cells, hepatocytes and bile ductular structures in patients with fulminant liver failure. However, the role of OPN in ACLF is yet to be elucidated. Therefore, the current prospective, observational study aimed to evaluate the value of OPN in the prediction of 90-day mortality in patients with ACLF.

\section{Materials and methods}

Patients and primary endpoint. Patients with HBV-ACLF $(n=54)$ or CHB $(n=81)$ were enrolled prospectively from January 2015 to June 2017 at the Department of Liver Diseases of the Third People's Hospital of Changzhou (Changzhou, China). Additionally, a total of 20 healthy individuals without HBV infection were recruited as healthy controls (HC). The demographic characteristics of all enrolled subjects are listed in Table I. The diagnosis of ACLF was based on previously described criteria (18), as follows: Acute hepatic insult manifested as jaundice (serum bilirubin $\geq 5 \mathrm{mg} / \mathrm{dl}$ or $85 \mu \mathrm{mol} / \mathrm{l}$ ) and coagulopathy [international normalized ratio (INR) $>1.5$ or prothrombin activity $<40 \%$ ], complicated within 4 weeks by ascites and/or encephalopathy in a patient with $\mathrm{CHB}$. $\mathrm{CHB}$ was diagnosed according to the Chinese guidelines for the prevention and treatment of CHB (2015 version) (19). Patients with autoimmune diseases, immunodeficiency disorders, alcoholic liver disease, liver cancer, drug-induced liver diseases, blood diseases or co-infection with other hepatitis viruses were excluded. Blood samples $(5 \mathrm{ml})$ were collected at the time of diagnosis of HBV-ACLF or CHB, centrifuged at $1,000 \mathrm{x} \mathrm{g}$ for $10 \mathrm{~min}$, and preserved at $-80^{\circ} \mathrm{C}$. Biochemical parameters were measured using an automatic analyzer (Hitachi 7600; Hitachi, Ltd., Tokyo, Japan) and coagulation parameters were determined using an STA Compact Max ${ }^{\circledR}$ analyzer (Diagnostica Stago, Inc., Parsippany, NJ, USA). Demographic and clinical data including sex, age, alanine transaminase (ALT), aspartate transaminase (AST), total bilirubin (TBil), creatinine, INR, serum HBV DNA, HBV serological markers, $\alpha$-fetoprotein (AFP) and blood cell counts were collected within the first $24 \mathrm{~h}$ after the diagnosis of HBV-ACLF. The end-point of the study was 90-day mortality or survival following the established diagnosis of ACLF.

The present study was anonymous, and the protocol was approved by the Ethics Committee of the Third People's Hospital of Changzhou according to the Declaration of Helsinki 2013. Written informed consent was obtained from all participants prior to the study.

ELISA. The serum concentrations of OPN were measured using a commercial ELISA kit (R\&D Systems, Inc., Minneapolis, MN, USA) according to the manufacturer's instructions. In brief, $50 \mu$ liluted (1:100) serum samples were added to the ELISA plates pre-coated with a captured OPN antibody, absorbance was measured at $450 \mathrm{~nm}$ using an ELISA plate reader (Synergy HT; BioTek Instruments, Inc., Winooski, VT, USA), and a duplicate assay was performed; consequently, a standard curve was generated.

Score systems. MELD (4) and MELD-Na (5) scores were calculated using the formulas described previously,

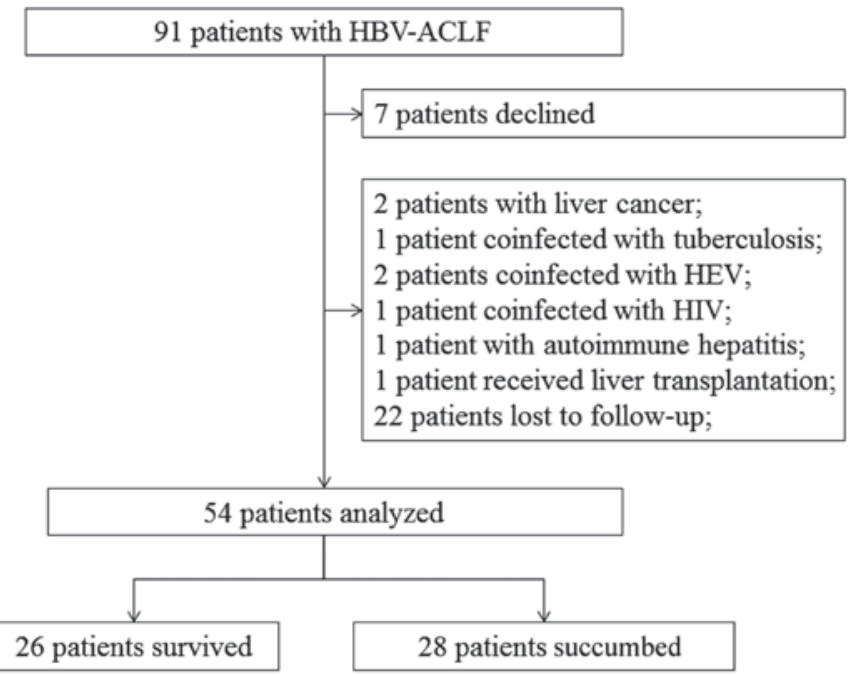

Figure 1. Selection and outcomes of patients with HBV-ACLF. HBV, hepatitis B virus; ACLF, acute-on-chronic liver failure; HEV, hepatitis E virus; HIV, human immunodeficiency virus.

as follows: MELD $=11.2 \times \ln (\mathrm{INR})+9.6 \times \ln$ [creatinine $(\mathrm{mg} / \mathrm{dl})]+3.8 \times \ln [$ total bilirubin $(\mathrm{TBil}, \mathrm{mg} / \mathrm{dl})]+6.4$; MELD-Na $=$ MELD + $1.59 \times(135-\mathrm{Na}+)$.

Statistical analysis. All data were analyzed using SPSS v17.0 (SPSS, Inc., Chicago, IL, USA). Continuous variables were expressed as the mean \pm standard deviation, and categorical values were expressed as frequencies. The differences in variables were analyzed using Student's t-test or one-way analysis of variance with Fisher's least significant difference post-hoc test (for normally distributed data), to determine the significance between the groups. The comparisons of frequencies were performed using the $\chi^{2}$ test. Pearson correlation analysis was used to evaluate correlations between OPN and other clinical indicators. Survival analysis was performed with Kaplan-Meier curves. Multivariate Cox proportional hazards regression was employed to analyze the independent risk factors of mortality. The optimal cut-off value of OPN and its sensitivity, specificity and positive and negative prediction values were predicted using SPSS Modeler 14.2 software (IBM Corp., Armonk, NY, USA). A two-sided $\mathrm{P}<0.05$ was considered to indicate a statistically significant difference.

\section{Results}

Characteristics of patients. The selection process and outcomes of the patients under study is illustrated in Fig. 1. A total of 54 patients with ACLF, who fulfilled the diagnostic criteria, were recruited prospectively. All of these patients received antiviral therapy at admission due to the positive HBV DNA level (>500 IU/ml). The demographic and clinical characteristics of all subjects in the ACLF, CHB and HC groups are listed in Table I. Patients with HBV-ACLF had elevated ALT, AST, TBil, creatinine and white blood cell (WBC) count compared with the $\mathrm{CHB}$ and $\mathrm{HC}$ groups $(\mathrm{P}<0.05)$. Notably, serum OPN level in the ACLF group was significantly higher than that in the $\mathrm{CHB}$ and $\mathrm{HC}$ groups (both $\mathrm{P}<0.01$; Fig. 2A). Furthermore, serum OPN in patients with $\mathrm{CHB}$ was elevated compared with 
Table I. Demographic and clinical characteristics of study subjects.

\begin{tabular}{|c|c|c|c|}
\hline \multirow[b]{2}{*}{ Variables } & \multicolumn{3}{|c|}{ Group } \\
\hline & HBV-ACLF $(n=54)$ & $\mathrm{CHB}(\mathrm{n}=81)$ & $\mathrm{HC}(\mathrm{n}=20)$ \\
\hline Age, years & $46.72 \pm 12.26^{\mathrm{a}}$ & $42.44 \pm 13.72$ & $34.49 \pm 10.11$ \\
\hline Male, $\mathrm{n}(\%)$ & $48(88.89)^{\mathrm{a}}$ & $64(79.01)$ & $13(65.00)$ \\
\hline HBeAg, positive rate, $\mathrm{n}(\%)$ & $10(18.52)$ & $21(25.93)$ & 0 \\
\hline ALT, U/1 & $480.81 \pm 604.13^{\mathrm{a}, \mathrm{b}}$ & $256.12 \pm 78.17^{\mathrm{a}}$ & $22.17 \pm 5.29$ \\
\hline AST, U/1 & $346.28 \pm 503.74^{\mathrm{a}, \mathrm{b}}$ & $210.13 \pm 46.77^{\mathrm{a}}$ & $16.22 \pm 8.30$ \\
\hline $\mathrm{TBil}, \mu \mathrm{mol} / 1$ & $305.06 \pm 154.82^{\mathrm{a}, \mathrm{b}}$ & $19.32 \pm 7.91$ & $12.19 \pm 4.51$ \\
\hline Creatinine, $\mu \mathrm{mol} / 1$ & $75.76 \pm 21.88^{\mathrm{a}, \mathrm{b}}$ & $61.19 \pm 16.33$ & $62.78 \pm 11.39$ \\
\hline INR & $2.33 \pm 0.95^{\mathrm{b}}$ & $1.40 \pm 0.32$ & N.D. \\
\hline AFP, $n g / m l$ & $348.31 \pm 641.87$ & N.D. & N.D. \\
\hline WBC count, E+09/1 & $8.28 \pm 4.60^{\mathrm{a}, \mathrm{b}}$ & $5.43 \pm 1.19$ & $4.26 \pm 1.63$ \\
\hline Neutrophil count, E+09/1 & $6.01 \pm 4.19^{\mathrm{a}, \mathrm{b}}$ & $3.11 \pm 0.78$ & $2.99 \pm 0.71$ \\
\hline Lymphocyte count, E+09/1 & $1.32 \pm 0.58$ & $0.89 \pm 0.23$ & $0.92 \pm 0.33$ \\
\hline Monocyte count, E+09/1 & $0.76 \pm 0.36^{\mathrm{a}, \mathrm{b}}$ & $0.46 \pm 0.37$ & $0.41 \pm 0.19$ \\
\hline Platelet count, E+09/1 & $90.19 \pm 51.57^{\mathrm{a}, \mathrm{b}}$ & $136.12 \pm 21.09$ & $155.19 \pm 33.10$ \\
\hline MELD & $23.07 \pm 5.79^{\mathrm{b}}$ & $5.28 \pm 2.17$ & N.D. \\
\hline MELD-Na & $21.65 \pm 11.24$ & N.D. & N.D. \\
\hline OPN, ng/ml & $8,490.96 \pm 3,750.30^{\mathrm{a}, \mathrm{b}}$ & $6,593.82 \pm 2,555.39^{a}$ & $3,880.36 \pm 1,644.27$ \\
\hline
\end{tabular}

Continuous data are expressed as means \pm standard deviation. ${ }^{\mathrm{a}} \mathrm{P}<0.05 \mathrm{vs}$. $\mathrm{HC}$; ${ }^{\mathrm{b}} \mathrm{P}<0.05$ vs. CHB; ACLF, acute-on-chronic liver failure; $\mathrm{CHB}$, chronic hepatitis B; HC, healthy controls; HBeAg, hepatitis B e antigen; ALT, alanine aminotransferase; AST, aspartate aminotransferase; TBil, total bilirubin; INR, international normalized ratio; AFP, $\alpha$-fetoprotein; WBC, white blood cell; MELD, model for end-stage liver disease; OPN, osteopontin; N.D., not determined.
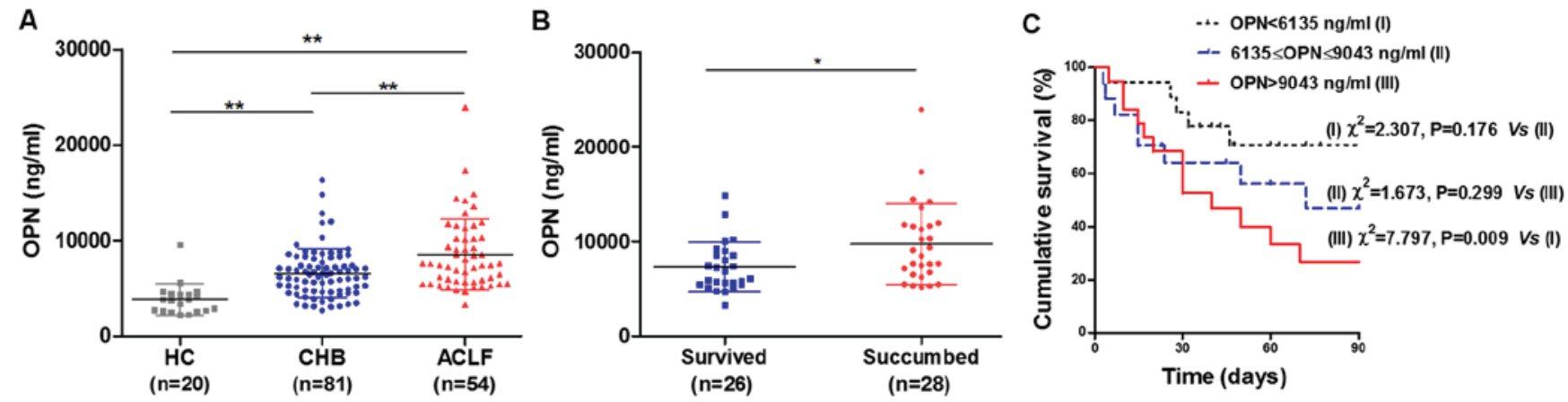

Figure 2. High OPN level predicts poor prognosis in HBV-ACLF. (A) Serum OPN in the ACLF group was significantly higher than that in the CHB and HC groups. (B) OPN in the non-survivor group was significantly higher when compared with the surviving patients. (C) Kaplan-Meier survival curves based on serum OPN to assess predictive value for 90-day mortality. The 90-day mortality rates were 27.78 (5/18), 52.94 (9/17) and 73.68\% (14/19) for the low $(\mathrm{OPN}<6,135 \mathrm{ng} / \mathrm{ml})$, intermediate $(6,135-9,043 \mathrm{ng} / \mathrm{ml})$ and high $(>9,043 \mathrm{ng} / \mathrm{ml})$ mortality risk groups, respectively, and those in the high risk group had a poorer prognosis compared with the low risk group $(\mathrm{P}=0.009)$. $\mathrm{P}<0.05$ and ${ }^{* *} \mathrm{P}<0.01$ as indicated. OPN, osteopontin; ACLF, acute-on-chronic liver failure; $\mathrm{CHB}$, chronic hepatitis B; HC, healthy controls.

that in the $\mathrm{HC}$ group $(\mathrm{P}<0.01)$. Subsequently, the ACLF patients were divided into cirrhotic and non-cirrhotic subgroups based on ultrasonography at admission, and no significant difference was identified in the OPN levels between the two subgroups ( $\mathrm{P}=0.973$; data not shown).

OPN in surviving and non-surviving patients with ACLF. Among the 54 patients with ACLF, 26 patients survived. No significant differences were observed in age, gender ratio or LC ratio between the surviving and non-surviving groups.
Significant differences were identified regarding TBil, INR, lymphocytes, platelet count, MELD, and MELD-Na score (Table II). Notably, OPN level in the non-surviving group was significantly higher than that in the surviving group ( $\mathrm{P}<0.05$; Fig. $2 \mathrm{~B}$ and Table II). Multivariable Cox regression analysis indicated that MELD $[\mathrm{P}=0.004$, odds ratio $(\mathrm{OR})=1.112,95 \%$ confidence interval $(\mathrm{CI}): 1.021-1.148]$ and OPN $(\mathrm{P}=0.021, \mathrm{OR}=1.104,95 \% \mathrm{CI}: 1.003-1.116)$ were independent risk factors for 90-day mortality (data not shown). 
Table II. Comparison of the surviving and non-surviving patients with HBV-ACLF.

\begin{tabular}{|c|c|c|c|}
\hline Variables & Surviving $(n=26)$ & Non-surviving $(\mathrm{n}=28)$ & P-value \\
\hline Age, years & $44.65 \pm 13.14$ & $48.64 \pm 11.28$ & 0.236 \\
\hline Male, n (\%) & $22(84.62)$ & $26(92.86)$ & 0.299 \\
\hline Cirrhosis, n (\%) & $14(53.85)$ & $18(64.29)$ & 0.435 \\
\hline HBeAg, positive rate, $\mathrm{n}(\%)$ & $5(19.23)$ & $5(17.86)$ & 0.586 \\
\hline ALT, U/1 & $422.96 \pm 592.98$ & $534.54 \pm 620.19$ & 0.503 \\
\hline $\mathrm{AST}, \mathrm{U} / 1$ & $290.65 \pm 409.43$ & $397.93 \pm 580.65$ & 0.440 \\
\hline $\mathrm{TBil}, \mu \mathrm{mol} / 1$ & $231.48 \pm 112.32$ & $373.38 \pm 159.10$ & $<0.001$ \\
\hline Creatinine, $\mu \mathrm{mol} / 1$ & $79.22 \pm 21.97$ & $72.56 \pm 21.69$ & 0.268 \\
\hline INR & $1.89 \pm 0.53$ & $2.72 \pm 1.08$ & 0.001 \\
\hline $\mathrm{AFP}, \mathrm{ng} / \mathrm{ml}$ & $310.90 \pm 388.93$ & $383.04 \pm 816.13$ & 0.684 \\
\hline WBC count, E+09/1 & $7.59 \pm 4.59$ & $8.91 \pm 4.59$ & 0.296 \\
\hline Neutrophil count, E+09/1 & $5.31 \pm 4.18$ & $6.84 \pm 4.13$ & 0.181 \\
\hline Lymphocyte count, E+09/1 & $1.54 \pm 0.66$ & $1.12 \pm 0.41$ & 0.017 \\
\hline Monocyte count, E+09/1 & $0.67 \pm 0.28$ & $0.85 \pm 0.40$ & 0.062 \\
\hline Platelet count, E+09/1 & $105.65 \pm 61.28$ & $75.82 \pm 36.06$ & 0.032 \\
\hline MELD & $20.39 \pm 5.19$ & $25.55 \pm 5.25$ & 0.001 \\
\hline MELD-Na & $17.02 \pm 8.91$ & $25.94 \pm 11.61$ & 0.003 \\
\hline OPN, ng/ml & $7,283.32 \pm 2,638.39$ & $9,730.02 \pm 4,276.14$ & 0.015 \\
\hline
\end{tabular}

Continuous data are expressed as means \pm standard deviation. Student's t-test and $\chi^{2}$ test were used for comparisons as appropriate. ACLF, acute-on-chronic liver failure; HBeAg, hepatitis B e antigen; ALT, alanine aminotransferase; AST, aspartate aminotransferase; TBil, total bilirubin; INR, international normalized ratio; AFP, $\alpha$-fetoprotein; WBC, white blood cell; MELD, model for end-stage liver disease; OPN, osteopontin.
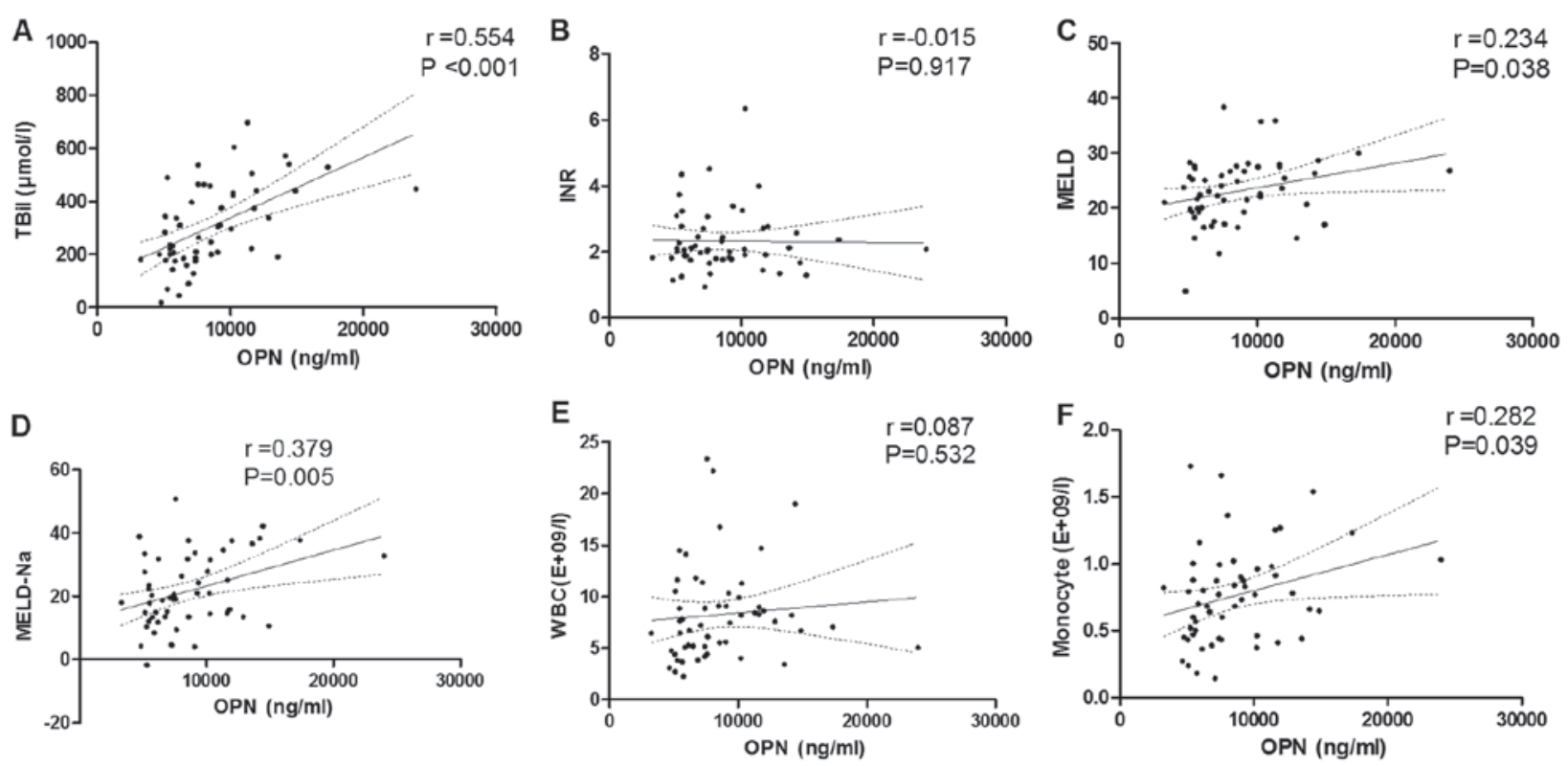

Figure 3. Higher OPN level indicates liver injury in ACLF. OPN levels in serum were positively correlated with (A) serum TBil but not associated with (B) plasma INR value. A significant positive correlation was observed between OPN and (C) MELD, as well as (D) MELD-Na. Serum OPN did not correlate with (E) WBC count, but positively correlated with (F) monocyte count in peripheral blood. OPN, osteopontin; TBil, total bilirubin; INR, international normalized ratio; MELD, model for end-stage liver disease; WBC, white blood cell; r, correlation coefficient.

The ACLF patients were stratified into three groups according to serum OPN levels (low mortality risk: $<6,135 \mathrm{ng} / \mathrm{ml}$; intermediate risk: $6,135-9,043 \mathrm{ng} / \mathrm{ml}$; and high risk: >9,043 ng/ml), predicted using SPSS Modeler 14.2 software. For the low, intermediate and high risk groups, the 90-day mortality rate was 27.78 (5/18), 52.94 (9/17) and $73.68 \%$ (14/19), respectively, and those in the high risk group had a poorer prognosis compared with the low risk group ( $\mathrm{P}=0.009$; Fig. $2 \mathrm{C})$. With an optimal cut-off value of $9,043 \mathrm{ng} / \mathrm{ml}$, OPN achieved $50.00 \%$ sensitivity, 
$80.77 \%$ specificity, $73.68 \%$ positive prediction value and $60.00 \%$ negative predictive value regarding prediction of high mortality risk.

Correlations between OPN and liver injury indicators. TBil, INR, MELD and MELD-Na scores were indicated as markers of liver injury severity in ACLF patients (Table II); thus, the correlations of OPN with these indicators were determined. Serum OPN level was positively correlated with serum TBil $(\mathrm{r}=0.554, \mathrm{P}<0.001$; Fig. 3A), though not with plasma INR value (Fig. 3B). A significant positive correlation was observed between OPN and MELD score ( $\mathrm{r}=0.234, \mathrm{P}=0.038$; Fig. 3C), as well as MELD-Na score ( $r=0.379, \mathrm{P}=0.005$; Fig. 3D). Although OPN was not associated with WBC count (Fig. 3E), it was positively correlated with monocyte count in peripheral blood ( $\mathrm{r}=0.282$, $\mathrm{P}=0.039$; Fig. 3F). AFP level and neutrophil, lymphocyte and platelet counts were not significantly correlated with OPN (data not shown).

\section{Discussion}

In the present study, it was demonstrated that OPN was significantly higher in HBV-ACLF patients, particularly in those who succumbed to mortality. Additionally, OPN was positively correlated with TBil level, MELD and MELD-Na score, and was implicated as an independent risk factor for prognosis of ACLF. Furthermore, OPN $>9,043 \mathrm{ng} / \mathrm{ml}$ predicted a poor prognosis.

As described previously, ACLF is a syndrome associated with a high rate of short-term mortality, and patients are typically diagnosed at a late stage when effective treatments are not applicable except for liver transplantation $(1,3)$. Considering the high mortality rate, it is important to identify those at high risk of mortality and refer these patients for liver transplantation. While evaluation of ACLF patients is recommended, the procedure is not optimal, owing to inadequate criteria. Recently, Graupera et al (20) reported that OPN levels were associated with the 3-month probability of readmission in patients with liver cirrhosis; thus, the current study hypothesized and validated that OPN may be associated with the exacerbation of CHB or LC. Serum OPN level was higher in ACLF patients, and was positively correlated with MELD and MELD-Na scores. According to the survival analysis, ACLF patients with high OPN level $(>9,043 \mathrm{ng} / \mathrm{ml})$ should be referred for liver transplantation at the earliest possible time, due to the high 90-day mortality rate. Collectively, these results suggested that OPN and MELD were complementary for the prediction of prognosis in ACLF patients.

OPN is involved in cell migration and inflammatory signaling, and previous studies have demonstrated that OPN was associated with hepatic inflammation and invasiveness in hepatocellular carcinoma (HCC) $(8,9,21)$. In line with these experimental observations, an association of circulating OPN levels and HCC incidence has previously been demonstrated in a population cohort of more than 520,000 subjects from ten Western European countries (21). As cell proliferation is among the key mechanisms in HCC and liver repair $(8,22-24)$, high levels of OPN may suggest tumor progression or liver regeneration. However, the present study demonstrated that high levels of OPN predicted poor prognosis in ACLF. Therefore, it would be premature to conclude that OPN is protective or beneficial to liver regeneration in ACLF patients.

Furthermore, Urtasun et al (25) demonstrated that OPN was expressed by hepatic stellate cells (HSCs), and upregulated collagen I production in oxidant stress, ultimately leading to fibrogenesis. Wang et al (26) identified a role of OPN in HPC activation, and demonstrated that HPC-driven ductular reaction was a critical mechanism underlying the progression of liver fibrosis. Another study demonstrated that OPN neutralization suppressed liver fibrosis in mice (27). As a compensatory mechanism of liver injury repair, liver fibrosis is exacerbated in $\operatorname{ACLF}(27,28)$; thus, OPN may be a potential therapeutic target during ACLF development. Additionally, Coombes et al (27) indicated a role of OPN in HPC expansion and OPN deficiency, which reduced the response of HPCs, thereby affecting the proliferation ability of hepatocytes. However, downregulation of OPN may inhibit liver regeneration, which is critical for the recovery of liver failure. Thus, further studies are required to identify the role of OPN in experimental models of liver failure.

Previous studies have demonstrated that two forms of OPN coexist: An intracellular form (iOPN) and a secreted form (sOPN) $(15,29,30)$. Although sOPN was measured in the present study, further studies are imperative to investigate the role of iOPN in lymphocytes and dendritic cells. Furthermore, as sOPN may be produced by many cell types, including hepatocytes, cholangiocytes, HSCs, macrophages, T lymphocytes and natural killer $\mathrm{T}$ cells, identifying the primary sources of OPN in liver failure is challenging $(6,28,31)$. In the present study, Pearson's correlation analysis indicated that OPN levels were not associated with WBC or lymphocyte count; however, a positive correlation was established with monocyte count in the peripheral blood. This result suggests that monocytes may contribute to the production of sOPN in ACLF patients. Thus, immunohistochemistry may be useful for investigating the role iOPN and the origin of sOPN in future studies.

Nonetheless, the present study had several limitations. First, although OPN was associated with prognosis in ACLF, the mechanisms underlying the role of OPN were not investigated. Second, the dynamic changes in serum OPN should be determined during the progression of ACLF, along with an evaluation of long-term prognostic value. Finally, multicenter studies are also required for confirmation of the current findings.

In conclusion, serum OPN was significantly higher in patients with ACLF and correlated with indicators of liver injury. Additionally, it was an independent risk factor associated with prognosis. Collectively the present findings suggest that OPN may be a predictor of prognosis in patients with HBV-ACLF.

\section{Acknowledgements}

The present study was supported by the Chinese Foundation for Hepatitis Prevention and Control-Tianqing Liver Disease Research Fund Subject (grant nos. TQGB201700139 and TQGB20150006), the Major Projects of Changzhou Municipal Health and Family Planning Commission (grant no.ZD2011002) and the Projects of Changzhou Science and Technology 
Commission (grant no. CJ20160024). Yuan Xue conceived and designed the study. Longgen Liu and Jianchun Lu performed the experiments. Chunyan Ye, Lin Lin, Shuqin Zheng, Hongyu Zhang and Qing Lan collected and confirmed the data. Yuan Xue, Longgen Liu and Jianchun Lu performed the analysis and drafted the manuscript. All authors read and approved the final manuscript.

\section{References}

1. Hernaez R, Solà E, Moreau R and Ginès P: Acute-on-chronic liver failure: An update. Gut 66: 541-553, 2017.

2. Arroyo V and Jalan R: Acute-on-chronic liver failure: Definition, diagnosis, and clinical characteristics. Semin Liver Dis 36: 109-116, 2016.

3. Arroyo V and Moreau R: Diagnosis and prognosis of acute on chronic liver failure (ACLF) in cirrhosis. J Hepatol 66: 451-453, 2017.

4. Kamath PS, Wiesner RH, Malinchoc M,Kremers W, Therneau TM, Kosberg CL, D'Amico G, Dickson ER and Kim WR: A model to predict survival in patients with end-stage liver disease. Hepatology 33: 464-470, 2001.

5. Biggins SW, Kim WR, Terrault NA, Saab S, Balan V, Schiano T, Benson J, Therneau T, Kremers W, Wiesner R, et al Evidence-based incorporation of serum sodium concentration into MELD. Gastroenterology 130: 1652-1660, 2006.

6. Diao H, Iwabuchi K, Li L, Onoe K, Van Kaer L, Kon S, Saito Y, Morimoto J, Denhardt DT, Rittling S, et al: Osteopontin regulates development and function of invariant natural killer T cells. Proc Natl Acad Sci USA 105: 15884-15889, 2008.

7. Diao H, Kon S, Iwabuchi K, Kimura C, Morimoto J, Ito D, Segawa T, Maeda M, Hamuro J, Nakayama T, et al: Osteopontin as a mediator of NKT cell function in T cell-mediated liver diseases. Immunity 21: 539-550, 2004.

8. Pereira TA, Syn WK, Pereira FE, Lambertucci JR, Secor WE and Diehl AM: Serum osteopontin is a biomarker of severe fibrosis and portal hypertension in human and murine schistosomiasis mansoni. Int J Parasitol 46: 829-832, 2016.

9. Matsue Y, Tsutsumi M, Hayashi N, Saito T, Tsuchishima M, Toshikuni N, Arisawa T and George J: Serum osteopontin predicts degree of hepatic fibrosis and serves as a biomarker in patients with hepatitis C virus infection. PLoS One 10: e0118744, 2015.

10. Zhao L, Li T, Wang Y, Pan Y, Ning H, Hui X, Xie H, Wang J, Han Y, Liu Z, et al: Elevated plasma osteopontin level is predictive of cirrhosis in patients with hepatitis B infection. Int J Clin Pract 62: 1056-1062, 2008.

11. Fan X, He C, Jing W, Zhou X, Chen R, Cao L, Zhu M, Jia R, Wang H, Guo Y, et al: Intracellular Osteopontin inhibits toll-like receptor signaling and impedes liver carcinogenesis. Cancer Res 75: 86-97, 2015 .

12. Patouraux S, Rousseau D, Rubio A, Bonnafous S, Lavallard VJ, Lauron J, Saint-Paul MC, Bailly-Maitre B, Tran A, Crenesse D, et al: Osteopontin deficiency aggravates hepatic injury induced by ischemia-reperfusion in mice. Cell Death Dis 5: e1208, 2014.

13. Liu Y, Cao L, Chen R, Zhou X, Fan X, Liang Y, Jia R, Wang H, Liu G, Guo Y, et al: Osteopontin Promotes Hepatic Progenitor Cell Expansion and Tumorigenicity via Activation of $\beta$-Catenin in Mice. Stem Cells 33: 3569-3580, 2015.

14. Lee SH, Park JW, Woo SH, Go DM, Kwon HJ, Jang JJ and Kim DY: Suppression of osteopontin inhibits chemically induced hepatic carcinogenesis by induction of apoptosis in mice. Oncotarget 7: 87219-87231, 2016.
15. Srungaram P, Rule JA, Yuan HJ, Reimold A, Dahl B, Sanders C and Lee WM: Acute Liver Failure Study Group: Plasma osteopontin in acute liver failure. Cytokine 73: 270-276, 2015.

16. Tajiri T, Tate G, Kunimura T, Endo Y, Inoue K, Mitsuya T, Morohoshi $\mathrm{T}$ and Yoshiba M: Osteopontin expression in proliferated bile ductules: The correlation with liver damage in fulminant hepatitis. Dig Dis Sci 50: 188-195, 2005.

17. Arai M, Yokosuka O, Kanda T, Fukai K, Imazeki F, Muramatsu M, Seki N, Miyazaki M, Ochiai T, Hirasawa H, et al: Serum osteopontin levels in patients with acute liver dysfunction. Scand J Gastroenterol 41: 102-110, 2006.

18. Sarin SK, Kedarisetty CK, Abbas Z, Amarapurkar D, Bihari C, Chan AC, Chawla YK, Dokmeci AK, Garg H, Ghazinyan H, et al: APASL ACLF Working Party: Acute-on-chronic liver failure: Consensus recommendations of the Asian Pacific Association for the Study of the Liver (APASL) 2014. Hepatol Int 8: 453-471, 2014.

19. Tang CM, Yau TO and Yu J: Management of chronic hepatitis B infection: Current treatment guidelines, challenges, and new developments. World J Gastroenterol 20: 6262-6278, 2014.

20. Graupera I, Solà E, Fabrellas N, Moreira R, Solé C, Huelin P, de la Prada G, Pose E, Ariza X, Risso A, et al: Urine Monocyte Chemoattractant Protein-1 Is an Independent Predictive Factor of Hospital Readmission and Survival in Cirrhosis. PLoS One 11: e0157371, 2016.

21. Duarte-Salles T, Misra S, Stepien M, Plymoth A, Muller D, Overvad K, Olsen A, Tjønneland A, Baglietto L, Severi G, et al: Circulating osteopontin and prediction of hepatocellular carcinoma development in a large European population. Cancer Prev Res (Phila) 9: 758-765, 2016.

22. Rastogi A, Bihari C, Maiwall R, Ahuja A, Sharma MK, Kumar A and Sarin SK: Hepatic stellate cells are involved in the pathogenesis of acute-on-chronic liver failure (ACLF). Virchows Arch 461: 393-398, 2012.

23. Shih SC, Ho TC, Chen SL and Tsao YP: Pigment epithelium-derived factor (PEDF) peptide promotes the expansion of hepatic stem/progenitor cells via ERK and STAT3-dependent signaling. Am J Transl Res 9: 1114-1126, 2017.

24. Wang G, Zhao C, Chen S, Li X, Zhang L, Chang C and Xu C: A preliminary in vivo study of the effects of OPN on rat liver regeneration induced by partial hepatectomy. Mol Biol Rep 43: 1371-1382, 2016

25. Urtasun R, Lopategi A, George J, Leung TM, Lu Y, Wang X, Ge X, Fiel MI and Nieto N: Osteopontin, an oxidant stress sensitive cytokine, up-regulates collagen-I via integrin $\alpha(\mathrm{V}) \beta(3)$ engagement and $\mathrm{PI} 3 \mathrm{~K} / \mathrm{pAkt} / \mathrm{NF} \mathrm{B}$ signaling. Hepatology 55: 594-608, 2012

26. Wang X, Lopategi A, Ge X, Lu Y, Kitamura N, Urtasun R, Leung TM, Fiel MI and Nieto N: Osteopontin induces ductular reaction contributing to liver fibrosis. Gut 63: 1805-1818, 2014.

27. Coombes JD, Swiderska-Syn M, Dollé L, Reid D, Eksteen B, Claridge L, Briones-Orta MA, Shetty S, Oo YH, Riva A, et al: Osteopontin neutralisation abrogates the liver progenitor cell response and fibrogenesis in mice. Gut 64: 1120-1131, 2015.

28. Strazzabosco M, Fabris L and Albano E: Osteopontin: A new player in regulating hepatic ductular reaction and hepatic progenitor cell responses during chronic liver injury. Gut 63: 1693-1694, 2014.

29. Uede T: Osteopontin, intrinsic tissue regulator of intractable inflammatory diseases. Pathol Int 61: 265-280, 2011.

30. Inoue M and Shinohara ML: Intracellular osteopontin (iOPN) and immunity. Immunol Res 49: 160-172, 2011.

31. Nagoshi S: Osteopontin: Versatile modulator of liver diseases. Hepatol Res 44: 22-30, 2014. 